\title{
Affective Videogames: The Problem of Wearability and Comfort
}

\author{
Andrea Bonarini ${ }^{1}$, Fiammetta Costa $^{2}$, Maurizio Garbarino ${ }^{1}$, Matteo Matteucci ${ }^{1}$, \\ Maximiliano Romero ${ }^{2}$, and Simone Tognetti ${ }^{1}$ \\ ${ }^{1}$ Department of Electronics and Information, \\ Politecnico di Milano \\ ${ }^{2}$ Health Care Design, Industrial Design Department (INDACO), \\ Politecnico di Milano \\ maximiliano.romero@polimi.it
}

\begin{abstract}
The aim of this paper is to report the interaction design process followed by an interdisciplinary team to develop an innovative ICT wearable device for affective video gaming. The process follows Norman and Draper's User Centered Design principles [1] including: functional development, laboratory test of the technology with human subjects, product design, prototype realization and experimentation with final users. The functioning of the device is based on the detection of physiological parameters, e.g., Blood Volume Pulse (BVP), Temperature (T), and Galvanic Skin Response (GSR), through electrodes placed on the forehead of the player. These signals are aimed at detecting the emotional state of the player by means of computational intelligence algorithms. This information can be used to modify the behavior of a videogame in order to maintain the player in the desired state of subjective enjoyment. Our goal was to develop a comfortable and easy to use device to avoid disturbs on the emotional state of the player.
\end{abstract}

Keywords: Design for wearability, Affective computing, Videogame.

\section{Introduction}

Videogames have seen recently a paradigm shift in user interaction; from passive devices such as joypad and keyboards they have moved to devices able to directly capture the player's intention from body movements and gesture [2]. This shift has shown how a strong relationship between game and user could be established through the use of devices improving the interaction. This aspect has often turned to be more important than perceptual realism of the game. To push this relationship further, we need to adapt the game to each single user monitoring her/his engagement and acting on the game parameters to keep her/his engaged all the time.

In this paper, we present a device to capture data to infer the user mood while interacting with a videogame. This device leverages on recent studies in affective computing that present computational intelligence algorithm for the detection of user preferences [3] and emotional state [4] from physiological signals such as Blood Volume Pulse (BVP), Temperature (T), and Galvanic Skin Response (GSR). Since 
the emotional state of the player may be affected by the presence of an invasive sensing device, we aimed at developing a comfortable and easy-to-use device.

The present work has been developed as collaboration between two research teams from the department of Electronics and Information and the department of Industrial Design of Politecnico di Milano. The first group, working in the Artificial Intelligence and Robotics Laboratory (AIRLab), has a background in electronic engineering and computational intelligence, while the Health Care Design (HCD) team is specialized in technological and user requirement integration.

While the design of the electronic back-end of the device is out of the scope of this paper, we focus here on the development process of the front-end hardware. This process had to face three main challenges; it should be easy to wear, it should not affect the interaction with the game, and it should provide reliable and artifact-free signals.

\section{Process Description and Motivations}

The technological feasibility of emotional detection from physiological signals in video gaming has been recently verified experimentally [3, 4]. The test, done on 75 volunteers, has shown how it is possible to establish a reliable relationship between physiological data and preference of the subject for a specific setting of the game with respect to alternative ones. The game used in the experiment was the TORCS car racing game [5], and the racing settings that have been compared were affecting the opponent's skills.

During the experiment, we have acquired a large number of physiological signals, and we have identified by statistical analysis those providing the larger amount of information needed to classify the emotional state. These have been acquired with a traditional data acquisition system, which requires the subject to be instrumented with sensors on fingers and chest, and these sensors are then wired to an acquisition device. However, by observing the subjects, we have noticed how the instrumentation made many subjects uneasy, possibly affecting both their performance and their emotional relationship with the game. Therefore, we have decided to design a new wearable interface that could be easily accepted by any player, and, at the same time, could provide enough information for emotion mapping.

Being both hands involved in the racing game (but this applies to other videogames as well) we have identified the forehead as a good candidate place to get the most interesting signals such as BVP, T and GSR. An acquisition system worn on the head makes it also possible to get signals from accelerometers and gyroscopes to monitor head movements, that can be useful to complement the physiological signals. The developed headset has been connected to the acquisition system via wireless communication.

On the market, there are some headsets with electrodes that have also been targeted to the videogame market (e.g., NIA by OCZ, Mindset by Neurosky, EPOC by Emotiv). However, they are mainly aimed at controlling the game through electric surface potentials while they are not aimed at detecting features that could be related to the affective state of the user such as BVP or GSR. 
To fill this market gap, three incremental steps have been performed regarding product design: the first step was to package the electronic components in a compact product, the second was to increase the wearability considering anthropometrical data [6] and pressure zones; the third concerns user acceptance considering aesthetic characteristics.

User comfort is not only related to the physiological condition, but psychological factors have also to be taken into account. In our case, this is particularly relevant because the goal is specifically to deduce psychological information rather than collect physiological data. Furthermore, the formal design of the packaging is important also for user acceptance since it should evoke the game world and not clinical instrumentation like most of the data acquisition devices available on the market. Several demonstrators have been produced with rapid prototyping techniques to test wearability and pleasure [7] with final users and achieve a satisfactory release.

\section{Design Process}

The AIRLab team initiated the present research but when the back-end has been sufficiently developed, it was necessary to focus on front end of the system. For this reason, the HCD group has been involved.

HCD group usually collaborate in multidisciplinary teams with ICT and Health Care experts and has developed a specific design process method that takes into account final users participation and co-design [8]. The aim of this method is to involve final users on the front-end side of the technological products from the early stage of the project in order to transfer User Experience to the back-end developers. Actually, if we consider a device as a complex product composed by Hardware and Software, final users might interact with both but they face mainly the front-end side. The back-end side involves engineering aspects and, normally, users are not aware of this layer.

This project was specifically focused on wearability and its impact on biosignals quality. Wearability is essential for a pleasant user experience of an affective videogame and it is also directly related to quality of biosignal acquisition depending on the sensors position and proper skin contact.

The back-end hardware is composed by two sensors that must be directly in contact with the skin, 2 Printed Circuit Board (PCB) and 1 battery. The initial effort was to reduce weight and dimensions and the second step was to decide the location of every component. According to Gemperle study [9], one of the main criteria for determining placement for dynamic wearability is to choose areas that have low movement and flexibility even when the body is in motion. We chose to locate the device on the head for two main reasons: all the needed signals could be collected and the head motion during videogaming was relatively low.

At the beginning, the internal tests have been realized with the prototype 1 (Fig. 1a. top left), when design team started to collaborate we developed the second prototype (Fig. 1b. top right) with rapid prototyping technique. After this, the backend hardware has been miniaturized and the third prototype has been developed (Fig. 1c. bottom left). At the end of the process has been developed the prototype 4, applied for the user test (Fig. 1d. bottom right) 


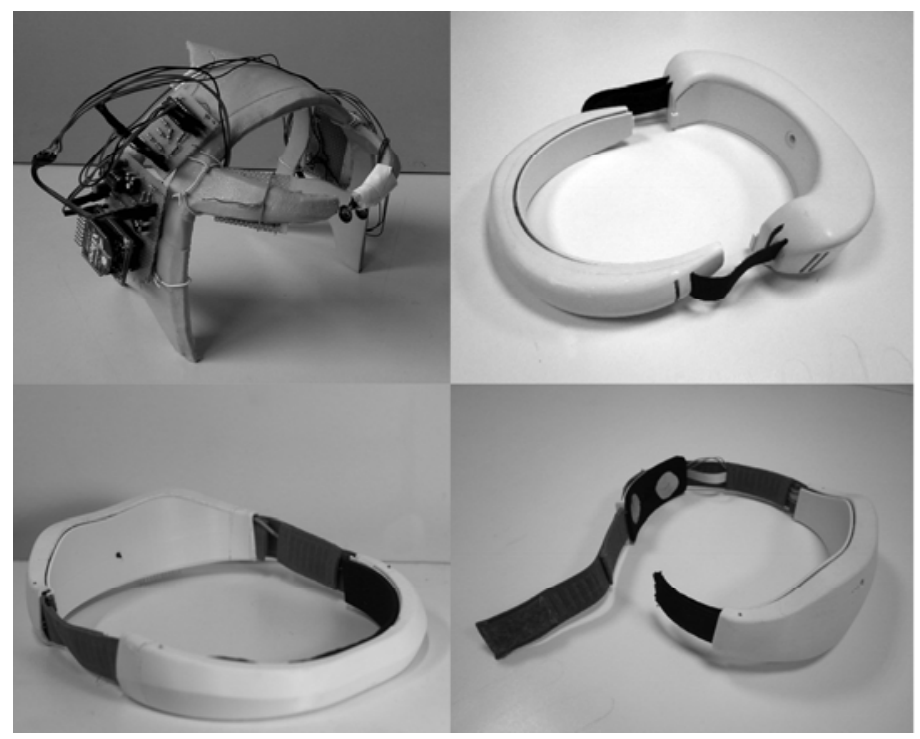

Fig. 1. Prototype evolution: (a) first prototype for laboratory tests, (b) designers' concept, (c) PCB miniaturization, (d) sensors contact improvements

Internal dimensions of front and back cases have been decided on the basis of literature anthropometrical data. Nevertheless, the variability of the head dimensions suggested to use fewer hard shapes and more elastic bands in order to allow the frontal case to become more flexible and improve the contact between biosensors and forehead.

\section{Instruments and Method}

The final test was performed in laboratory, by involving end users. We used the last prototype of our wearable device and a reference device, the Procomp Infiniti, for comparison. (Fig. 2.)

Our goal was to test wearability characteristics and quality of biosignals captured. For this reason, we decided a test protocol in which users were asked to perform different actions and, for each of them, we collected quantitative information. Users were not asked to play the videogame as we preferred them to concentrate on the feeling of wearability. The set up of the test included an empty desk with two separated boxes $80 \mathrm{~cm}$ far from the user. On the desk in front of the user our device and the reference device were located. One researcher has checked data transmission between device and computer and has taken note of time of performance, while the second has guided the users on the test.

During the test, users have been asked to dress the two devices (first the wearable device and then the reference one on the left hand), to stand still in a precise position (with both hands on the desk) and to perform the following series of movements: 


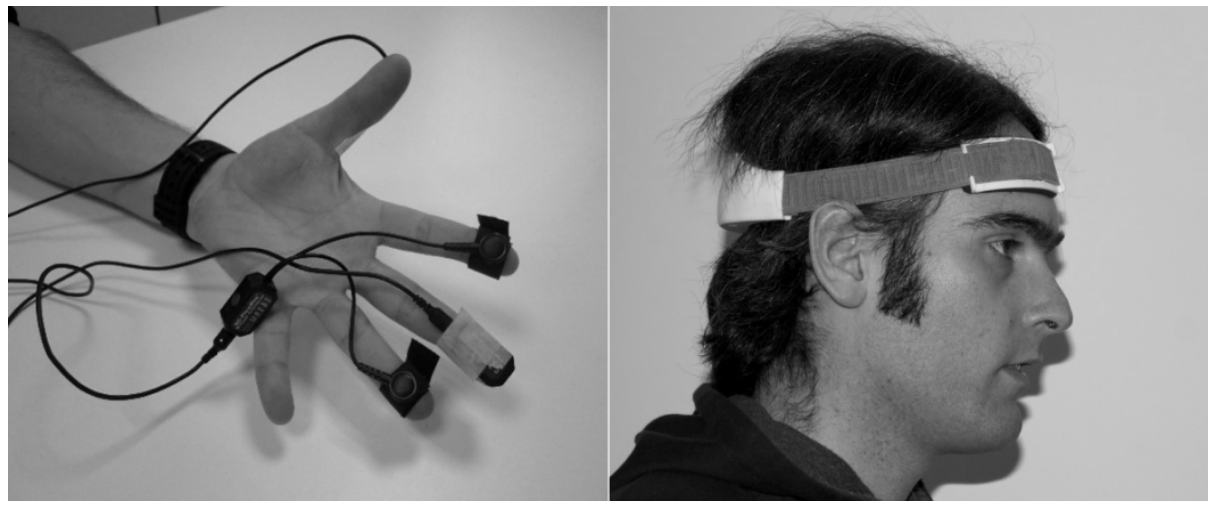

Fig. 2. Devices used for the final comparison test with user involvement: Procomp Infiniti hands sensors (left) and our last prototype of headset (right)

1. To reach the left box with right hand with natural movement (random motor strategy) and come back to neutral position.

2. To reach the right box with the left hand (sensorized hand) with natural movement (random motor strategy) and come back to neutral position.

3. To reach contemporaneously the right box with right hand and the left box with left hand, then come back to neutral position.

Finally, we ask them to remove the two devices (first the reference device and then the wearable one). At the end of the test we asked each user to fill a questionnaire with the following five questions:
a. Which device is easier to wear?
b. Which device is more immediate for the proper placement of the electrodes?
c. Which device is more immediate for the closure system?
d. Which device allows for more natural gestures?
e. Which device generates more sweat?

\section{Results}

We first present the analysis of questionnaires that qualitatively measure the wearablility of the devices from the user perspective; then we present a quantitative analysis of placement and removal times and a quantitative analysis of signal quality during both rest and movement periods.

\subsection{Questionnaire Analysis}

The questionnaire answer expresses the users preference, with respect to a given feature, between the wearable and reference device.

In Figure 3, histograms of the answers are reported for each question. All subjects reported that-our device can be worn more easily than the reference device(a). Less remarkable differences have been observed on the easiness by which people have understood how the device has to be positioned correctly; one user did not understand 
that the wearable device had to be placed on the head)(b). Users have perceived the closure system of the two devices equivalently complex(c); note that one of them reported a problem with our device related to the volume of the hair that made very difficult the closure of the system. All subjects agreed on the optimality of our device to allow natural movements (d). Finallly, most of the users reported that our device tended to increase the sweat activity(e).

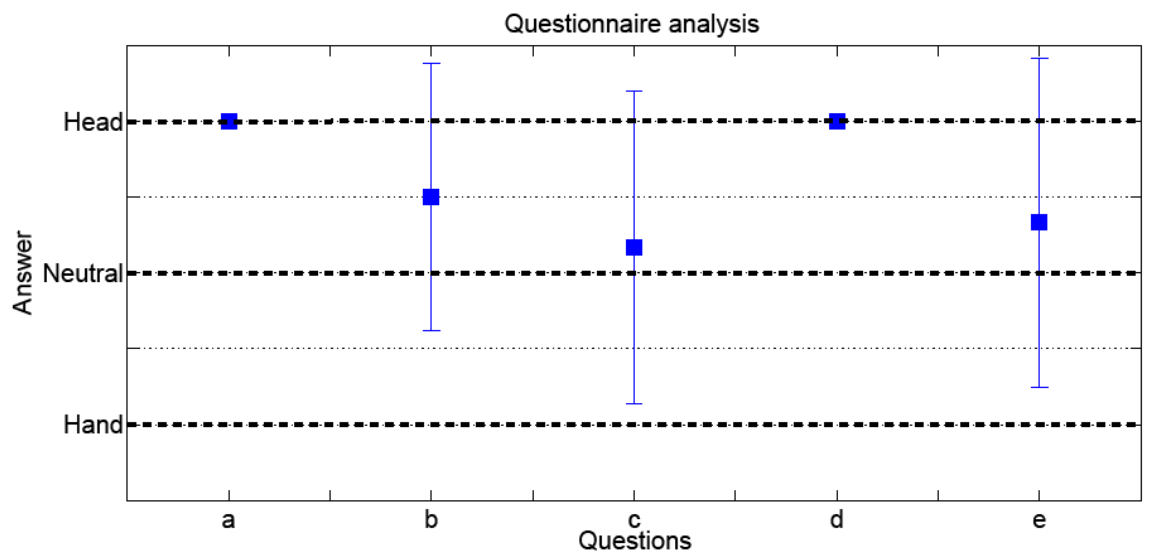

Fig. 3. Users' answers to usability and wearability questionnaire

Questions (a) and (d) confirm that our device we designed, meets the two fundamental objectives for an affective computing device of being easy-to-wear and to not compromise the standard interaction. Question (b) indicates that it is not so easy to understand how to correctly wear the headset. Question (c) indicates that the closure system has to be improved and finally question (e) indicates that our headset produces the side effect of increasing the sweat activity.

\subsection{Placement and Removal Times}

We measured the time required by a subject to correctly wear and unwear the devices. Each subject has been first shown how each device should be correctly placed. Note that both the devices have an equivalent number of physiological sensors. We are not considering the accelerometer that is present in our device while it is not available in the reference device. In Figure 4, the mean value and its confidence interval (with alpha $=0.05$ ) of placement and removal times for each devices are reported. Placement times are significantly higher for the reference device, positioned on the hand, compared to our wearable device (i.e., $12.58 \mathrm{~s}$ for the wearable device and $42.75 \mathrm{~s}$ for the reference one). A similar consideration holds for the removal times that have a mean value of $4.41 \mathrm{~s}$ of our device and $14.98 \mathrm{~s}$ the reference one.

Considering the assumption that the lower the placement/removal time is and the higher the easiness to wear is, this result confirms that we have satisfied the objective of building a device that is easy-to-wear. 


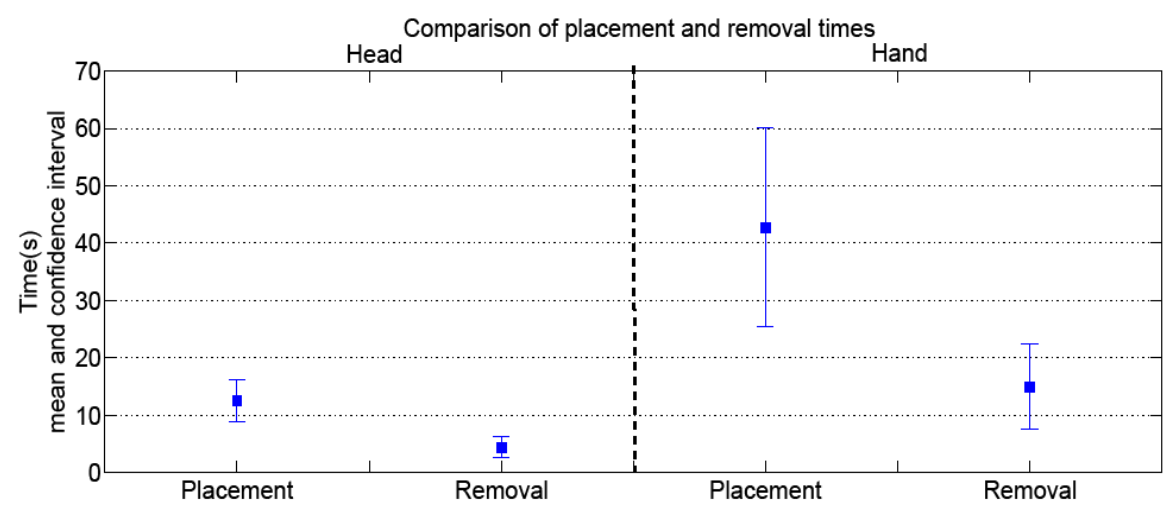

Fig. 4. Placement and removal times are significantly lower for our headset

\subsection{Signal Quality}

The last part of presented results concerns the quality of the signals obtained from our wearable device. Each wearable device, becoming more wearable tends to lower the signal quality since data are measured in suboptimal positions. However, even small movements could reduce the quality of the signal acquired by a standard medical device. Thus, in a natural environment we could accept to reduce the quality of signals by allowing the measurement even during movements.

In Figure 5, a comparison of BVP signals acquired by both devices during a resting period is presented. The signal from the forehead preserves almost all the intrinsic characteristics (i.e., waveform and positions of heart beats) with respect to a standard signal measured on the finger. A deeper analysis is required to understand whether the upper and lower envelop of BVP, representing the systolic and diastolic pressure, are also preserved.

In Figure 6 is presented a comparison of heart rate signals extracted from both segments of Figure 5. Note that the heart rate signals are very similar: there are only minimal differences that are due to the different position of detected heart beats. Such small differences do not decrease performances of an affective classifier.

In Figure 7 is presented a comparison of BVP signals acquired by both devices during a movement. In this situation, only the headset was able to measure continuously the signal without degradation. The reference device, instead, produced a signal that is not usable. The standard BVP signal is corrupted by the movement artifacts that, if not detected and removed, could lower the performances of an affective classifier [4].

To give an exhaustive analysis of BVP quality during the movements, we have manually evaluated each signal portion corresponding to a movement. We assigned them a quality value according to the percentage of signal that contains valid heart beats (i.e., the typical waveform is preserved). For instance, in the situation reported 
in Figure 7, our device has $100 \%$ quality, while the reference device has $0 \%$ quality. In Figure 8, the results of this analysis are reported. In particular for each movement (A) right hand, (B) left hand, (C) both hands, the mean value and confidence interval (with alpha=0.05) of signal quality are presented for both devices. We recall that signals have been acquired in parallel by both devices during each movement. Figure 8 shows that our device, placed on the head, measures the BVP signal with an overall quality of $80 \%$ during all the movements while the standard device, placed on the hand, produces a BVP with an overall quality of $20 \%$ during all the movements. It is interesting to notice that movement $\mathrm{C}$ produces a quality slightly higher than A and B. This is due to the fact that in movement A and B there is also head torsion.

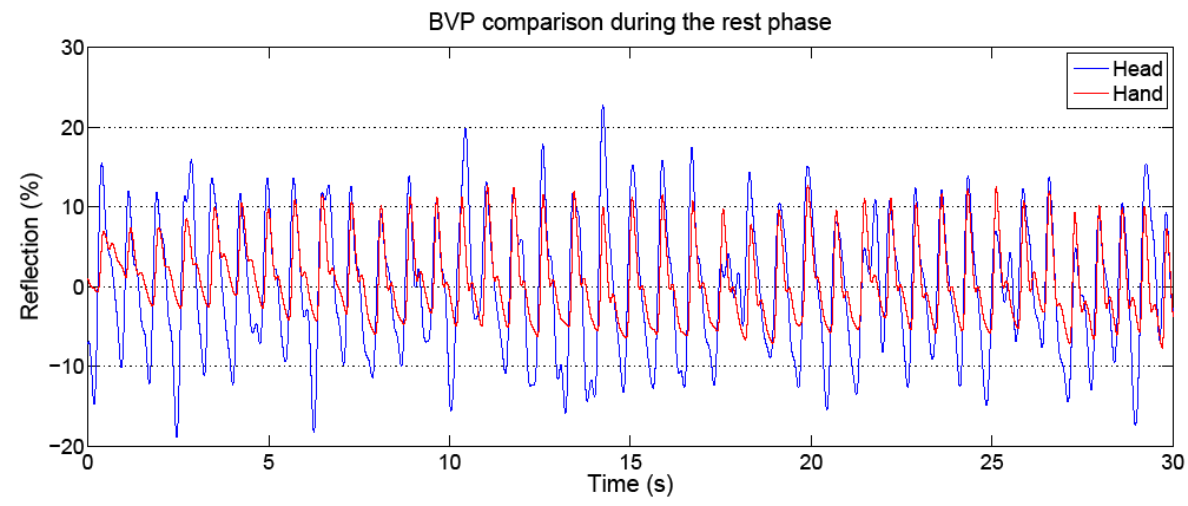

Fig. 5. Comparison of BVP signal acquired from finger and from forehead

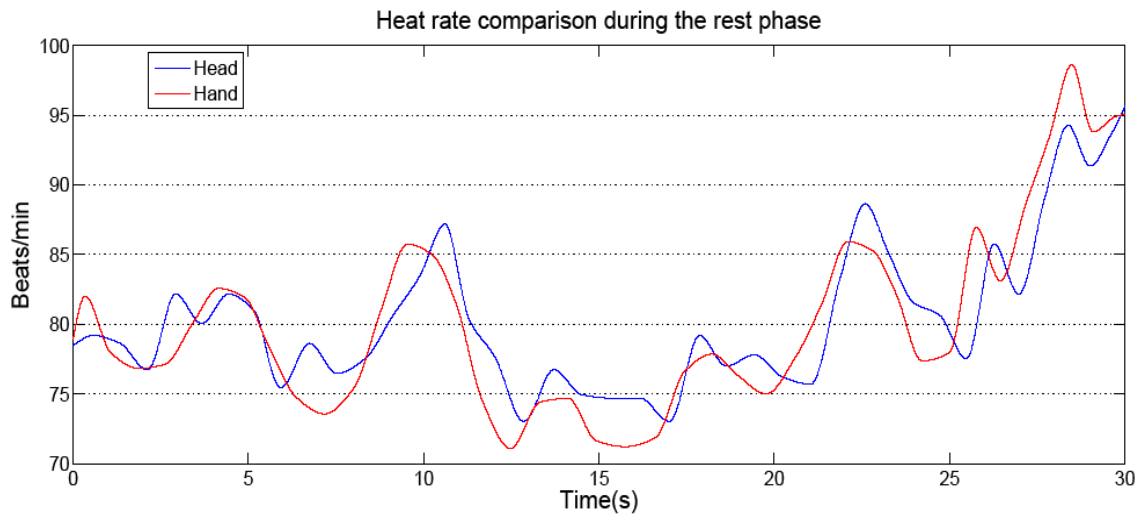

Fig. 6. Comparison of heart rates signal acquired from finger and from forehead 


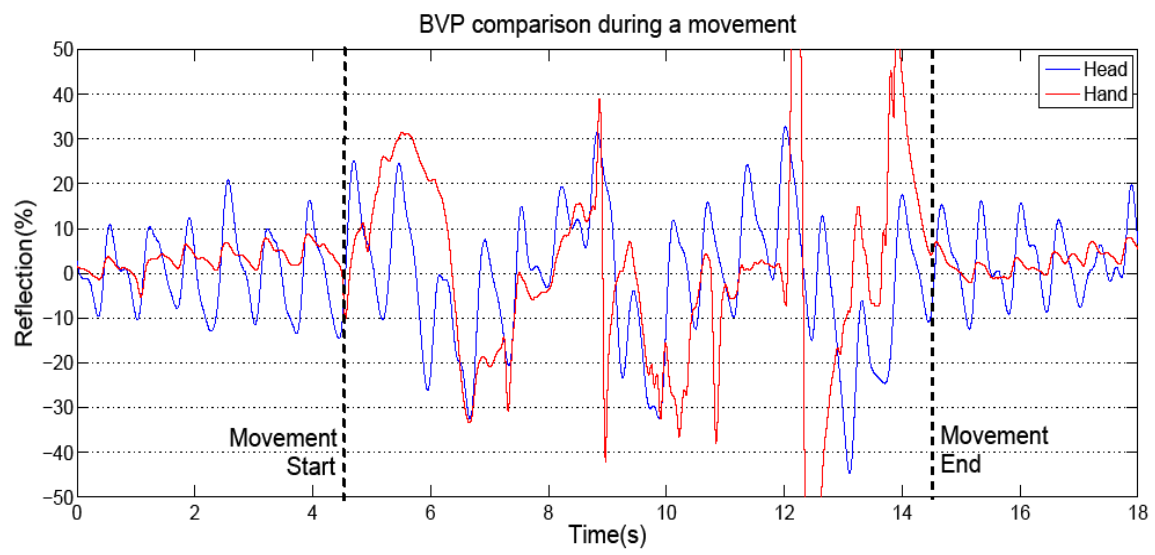

Fig. 7. Comparison of BVP signals during movements

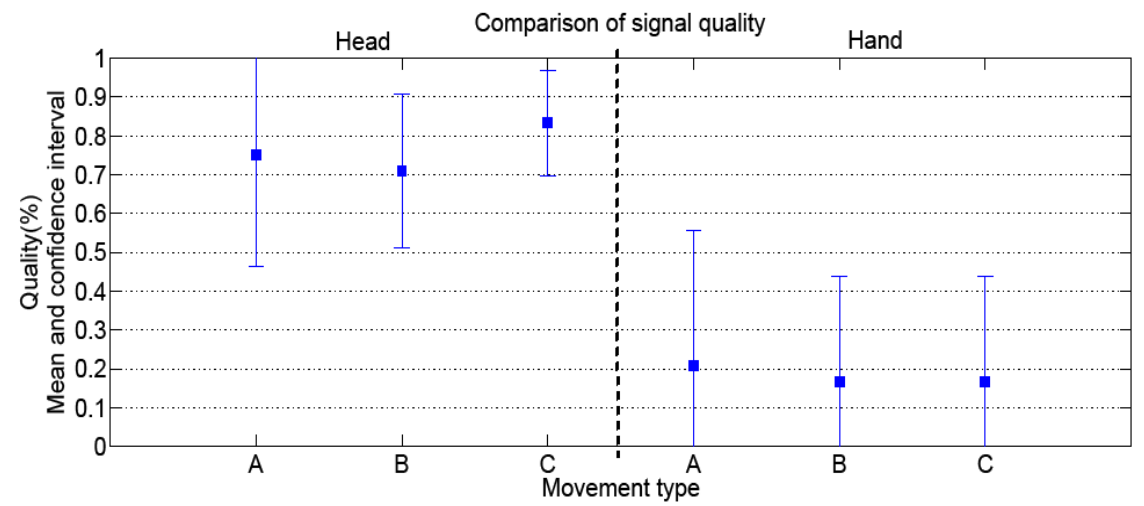

Fig. 8. Quality of signal during movements. Right hand (A), left hand (B), both hands (C)

This result demonstrated that the wearable device we designed acquires the BVP signal with a good quality during the movements that usually characterize activities in natural environments.

\section{Conclusion}

In this paper we presented the interaction design process followed by an interdisciplinary team to develop an innovative ICT wearable device for affective video gaming. The objective of this work was to design a wearable device that has the following requirements: it should be easy to wear, it should not affect the interaction with the game, and it should provide reliable and artifact-free signals.

The interaction design process we followed allowed us to better satisfy the device requirements. From one side, the HCD group, working of the front-end, aimed at satisfying the wearability aspects while the Artificial intellingence group, working on the back-end aimed at satisfying the signal quality aspects. 
The results we obtained demonstrate that the device requirement have been fairly satisfied. The questionnaires analysis shows that, from the user perspective, the wearable device is easy to wear and does not compromise the interaction. The placement/removal times analysis confirms that the wearability aspect has been met. Finally the signals analysis demonstrates that the wearable device produces better signals during a movement compared to a reference medical device.

Acknowledgments. We would like to thanks Paolo Villa, that designed the electronic hardware within his master thesis at Politecnico di Milano. We also thanks to Maicol Zoia, Marco Tonet and Davide Veronesi that help us to prototype the device.

\section{References}

1. Norman, D.A., Draper, S.W.: User centered system design: New perspectives on humancomputer interaction. Lawrence Erlbaum Associates, New Jersey (1986)

2. McArthur, V., Castellucci, S.J., MacKenzie, I.S.: An empirical comparison of "Wiimode" gun attachments for pointing tasks. In: Proceedings of the ACM Symposium on Engineering Interactive Computing Systems - EICS 2009, pp. 203-208. ACM, New York (2009)

3. Tognetti, S., Garbarino, M., Bonarini, A., Matteucci, M.: Modeling player enjoyment from physiological responses in a car racing game. In: Proceedings of IEEE International Conference on Computational Intelligence and Games (CIG), pp. 321-328. IEEE Computer Press, Los Alamitos (2010)

4. Tognetti, S., Garbarino, M., Bonanno, A.T., Matteucci, M., Bonarini, A.: Enjoyment Recognition From Physiological Data in a Car Racing Game. In: Proceedings of 3rd International Workshop on Affective Interaction in Natural Environments (AFFINE), pp. 1-6

5. The open racing car simulator website, http://torcs. sourceforge.net/

6. Peebles, L., Norris, B.: Adultdata: the handbook of adult anthropometric and strength measurements: data for design safety. Department of Trade and Industry, Government Consumer Safety Research, p. 294, London (1998)

7. Jordan, P.W.: Pleasure with Products: Beyond Usability. Taylor and Francis, Abington (2000)

8. Romero, M., Perego, P., Andreoni, G., Costa, F.: New Strategies for Technology Products Development in Health Care. In: MengJooEr (ed.) New Trends in Technologies: Control, Management, Computational Intelligence and Network Systems, Sciyo (2010); ISBN: 978-953-307-213-5

9. Gemperle, F., Kasabach, C., Stivoric, J., Bauer, M., Martin, R.: Design for wearability. In: Second International Symposium on Wearable Computers (Cat. No.98EX215), pp. 116-122., doi:10.1109/ISWC.1998.729537 